\title{
Advanced Uveal Melanoma
}

National Cancer Institute

\section{Source}

National Cancer Institute. Advanced Uveal Melanoma. NCI Thesaurus. Code C148515.

Uveal melanoma that has spread extensively to other anatomic sites or is no longer responding to treatment. 